\title{
A small vessel detection using a co-located multi-frequency FMCW MIMO radar
}

\author{
Suraya Zainuddin ${ }^{1}$, Nur Emileen Abd Rashid ${ }^{2}$, Idnin Pasya ${ }^{3}$, Raja Syamsul Azmir Raja Abdullah ${ }^{4}$, \\ Korhan Cengiz ${ }^{5}$ \\ ${ }^{1}$ Faculty of Electrical Engineering, Universiti Teknologi MARA, Malaysia \\ ${ }^{2,3}$ Microwave Research Institute, Universiti Teknologi MARA, Malaysia \\ ${ }^{1}$ Faculty of Electrical and Electronic Engineering Technology, Universiti Teknikal Malaysia Melaka, Malaysia \\ ${ }^{4}$ Faculty of Engineering, Universiti Putra Malaysia, Malaysia \\ ${ }^{5}$ Faculty of Engineering, Trakya University, Turkey
}

\section{Article Info}

Article history:

Received Mar 15, 2021

Revised Apr 8, 2021

Accepted Apr 26, 2021

\section{Keywords:}

FMCW

MIMO

Radar

Spectrum averaging

Swerling

\begin{abstract}
Small vessels detection is a known issue due to its low radar cross section (RCS). An existing shore-based vessel tracking radar is for long-distance commercial vessels detection. Meanwhile, a vessel-mounted radar system known for its reliability has a limitation due to its single radar coverage. The paper presented a co-located frequency modulated continuous waveform (FMCW) maritime radar for small vessel detection utilising a multiple-input multiple-output (MIMO) configuration. The radar behaviour is numerically simulated for detecting a Swerling 1 target which resembles small maritime's vessels. The simulated MIMO configuration comprised two transmitting and receiving nodes. The proposal is to utilize a multi-frequency FMCW MIMO configuration in a maritime environment by applying the spectrum averaging (SA) to fuse MIMO received signals for range and velocity estimation. The analysis was summarised and displayed in terms of estimation error performance, probability of error and average error. The simulation outcomes an improvement of $2.2 \mathrm{~dB}$ for a static target, and $0.1 \mathrm{~dB}$ for a moving target, in resulting the $20 \%$ probability of range error with the MIMO setup. A moving vessel's effect was observed to degrade the range error estimation performance between 0.6 to $2.7 \mathrm{~dB}$. Meanwhile, the proposed method was proven to improve the $20 \%$ probability of velocity error by $1.75 \mathrm{~dB}$. The impact of multi-frequency MIMO was also observed to produce better average error performance.
\end{abstract}

This is an open access article under the CC BY-SA license.

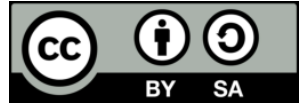

\section{Corresponding Author:}

Nur Emileen Abd Rashid

Microwave Research Institute, Universiti Teknologi MARA

40450 Shah Alam, Selangor, Malaysia

Email: emileen98@uitm.edu.my

\section{INTRODUCTION}

Detection of small vessels is crucial and one of the vital tasks in maritime radar. Various types of sea surface monitoring systems have been employed, and each has its pros and cons [1]. However, the known issue in maritime monitoring is that small vessel attributes reduce the probability of detection of modern radars, including ship-borne radar [2]. It is due to its low radar cross section (RCS) and low signal-to-noise ratio (SNR) [2]. A target's RCS depends on the radar's operating frequency, signal's incident angle, target's speed, material and geometry [3]. A small vessel such as an inshore fishing vessel produces RCS between 3 and $10 \mathrm{~m}^{2}$ at X-band [3], [4]. Small metal ships, fishing vessels and patrol vessels produce RCS up to 
$100 \mathrm{~m}^{2}$ at the similar band [3], [4]. These vessels fluctuation characteristic are normally categorised between slow and fast-fluctuating radar cross section (RCS) [5], [6], which leads to this paper discussion on a Swerling 1 maritime target behaviour. The recent trend of radar studies is working on the convergence of multiple approaches by exploiting each technique's quality to boost system performance.

In this work, a frequency modulated continuous wave (FMCW) radar topology which is known for its robustness towards noise and better resolution [7] was proposed by taking advantage of a $2 \times 2$ multipleinput multiple-output (MIMO) configuration. MIMO technique has been a recent interest due to its high capacity, increased diversity and interference suppression [8]. Thus, the MIMO configuration with multifrequency was proposed to be adopted for maritime target's estimation. Similar to all radar applications, the proposed configuration works based on the concept of signal reflection by a target of interest [9]-[12]. However, implementing the signal over MIMO requires orthogonality between FMCW signals as presented by Hinz et al. in their paper [13], which discussed the advantages and disadvantages of each FMCW MIMO approach. However, performance-wise of each system was not explained in the report. Some of the available FMCW MIMO schemes that have been studied by other researchers were time staggered [14], multifrequency [15]-[17] and different polarity [6]. Most of the multi-frequency researches were done with regards to utilisation of antenna array [15], [16], [18]. Reference [19] discussed the improvement brought by MIMO compared to the SISO configuration in detecting a small static vessel. A more extensive nodes configuration yields better target estimation and reduces range estimation error [19].

This paper presents a numerical simulation of a $2 \times 2$ MIMO radar which comprises two FMCW triangular waveform nodes applying a multi-frequency approach, in detecting a slow-fluctuating RCS static and doppler target. The multi-frequency approach was implemented in such the two co-located transmitting antennas operated at different frequencies with a frequency offset between sub-bands without antenna array implementation. Each transmitting signal was emitted by an independent antenna. Next, a spectrum averaging (SA) algorithm was applied to combine multiple received signals, and its performance was observed and analysed, in terms of probability of error and average error. The effect of the proposed configuration over a maritime target range estimation was explored, together with a moving vessel's impact.

\section{RESEARCH METHOD}

A multi-frequency approach was adopted to evaluate the proposed MIMO configuration for maritime target detection with interval band and SA scheme as per [19], through utilising a Monte Carlo simulation [20], [21]. The proposed transmitting signal is illustrated in Figure 1 . The $2 \times 2$ co-located MIMO FMCW consisted of two transmitting nodes that emit FMCW triangular signals, and two receiving nodes resided at the same site. The triangular sweep was opted due to its capability to obtain range and velocity with only one sweep [22], [23]. However, two sweeps were utilised in this paper to increase the resolution by having more number of samples [24]. The simulation started with baseband generators to produce the FMCW triangular chirps. In this case, there were two baseband generators with each constructed a baseband at a different frequency range, bandwidth 1 (BW1) and bandwidth 2 (BW2) as per Table 1. In the simulation, each baseband signal was modulated with the carrier signal to shift the baseband spectrum to a higher frequency of $1.3 \mathrm{GHz}$, suitable for long-range radar systems. It was simulated for 10,000 iterations and a $2 \mathrm{MHz}$ gap was applied in the case of interval band implementation by considering the filter order and filter delay.

The modulated signals were transmitted and propagated through a free-space path loss (FSPL) medium. Signals were reflected by a target which located $50 \mathrm{~m}$ from the radar. In the doppler target scenario, a simulation was carried out over a 60 knots target to resemble a motorboat [25]. Reflected signals were added with additive white Gaussian noise (AWGN) at the desired signal-to-noise ratio (SNR) before acquired by the MIMO receiver. Simplified diagram of the radar system is depicted in Figure 2. Table 1 summarises the numerical simulation properties of the setup.

Signals gathered by receivers were demodulated to shift the signal back to basebands. Demodulated signals were filtered by finite impulse response (FIR) bandpass filter with $100^{\text {th }}$ filter order utilising Hamming window to obtain a narrower transition band for MIMO sub-band processing. The 100th order introduces a filter delay of 50 for high isolation between MIMO sub-bands for better accuracy. A $2 \mathrm{MHz}$ interval band was applied for the separation to provide a sufficient gap when the received signal shifted in frequency domain due to doppler, not utilising too large bandwidth. A bandwidth utilisation may increase with the incremental MIMO configuration, sweep bandwidth and size of the interval. Besides, a smaller gap requires a higher filter order. Thus, the filter order selection needs to compensate for the narrow transition band and delay, a trade-off with the bandwidth requirement. Next, each filtered signal was mixed with the reference baseband signal to produce a beat signal, in the time domain. The beat signal was applied with the fast fourier transform (FFT) algorithm to convert to a frequency spectral before fused to SA block. As per Figure 1, there were four FFT outputs resulted from a $2 \times 2$ MIMO configuration. Peak detection was applied to the SA frequency spectral to determine beat and doppler frequencies, for range and velocity computation. 


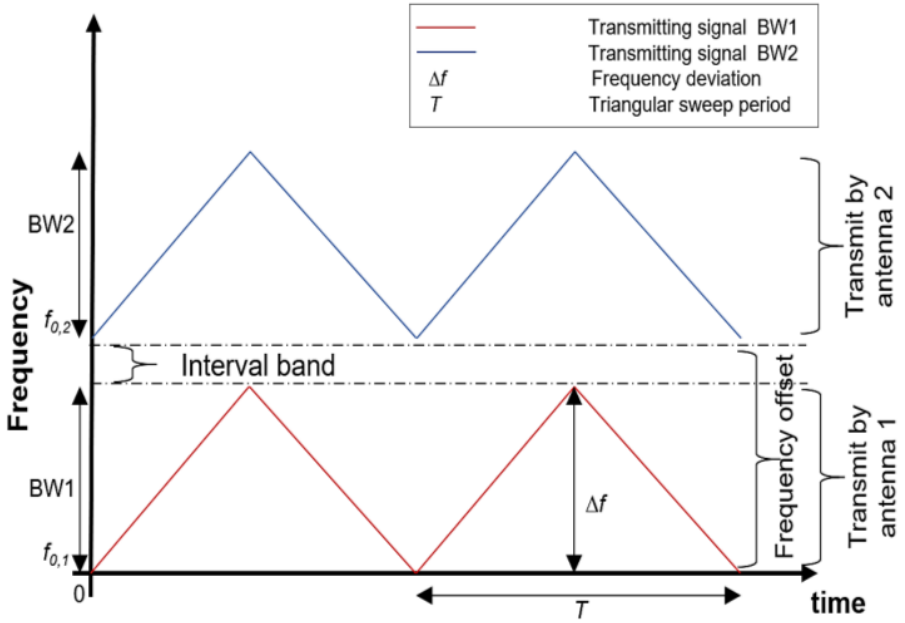

Figure 1. The proposed MIMO FMCW transmitting signals with frequency offset between sub-bands (with $2 \mathrm{MHz}$ gap)

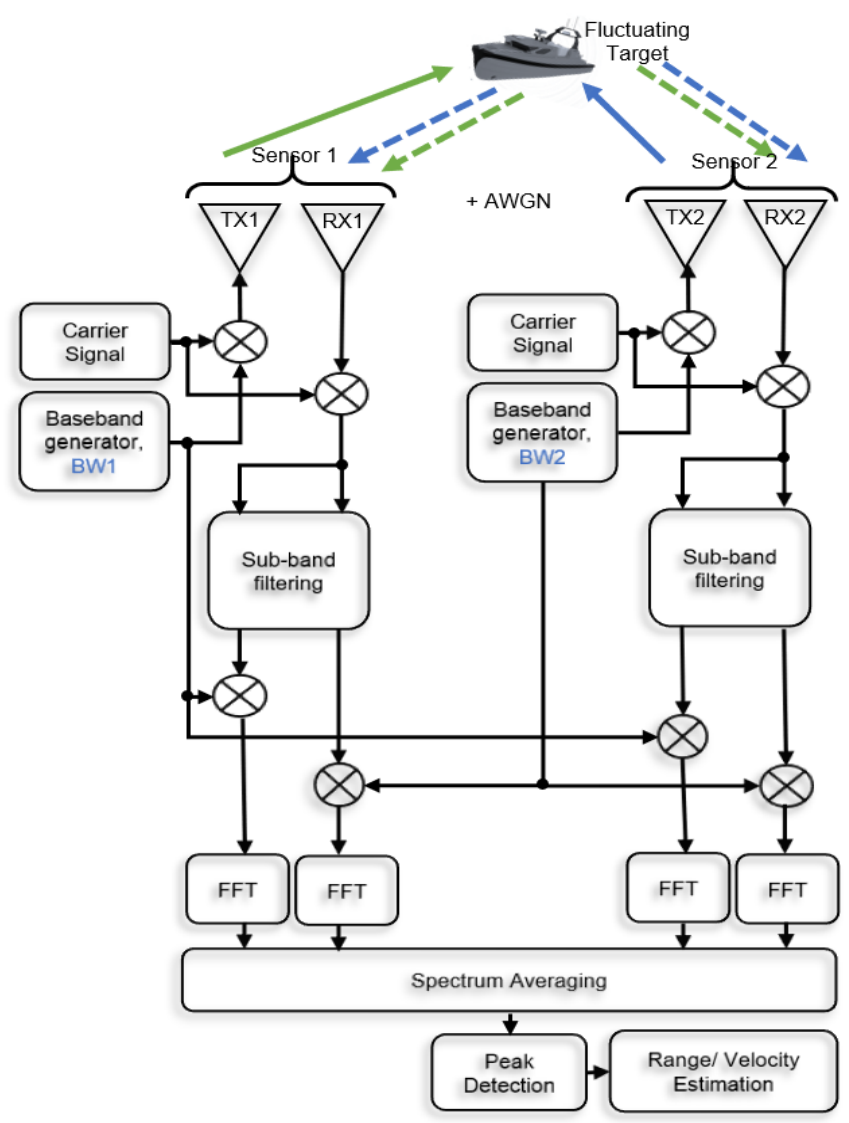

Figure 2. The proposed MIMO radar block diagram

Analysis was done to observe the radar performance in terms of probability of error and average error, for range and velocity. The probability of range error indicates the robustness of a system to produce accurate estimation. In contrast, the average error defines the accuracy of the measurement with the influence of additive white noise in a free-space path loss (FSPL) environment. In the case of a static target with radial velocity $=0$, beat frequency $\left(f_{b}\right)$ was sufficed to utilise the maximum peak in the requency spectrum's positive region as per Figure 3 . For a doppler target which radial velocity $\neq 0$, beat frequency $\left(f_{b}\right)$ and doppler 
frequency shift $\left(f_{d}\right)$ were calculated as per (1) and (2), respectively [26]. Figure 3 illustrates an example of a beat signal represented in the frequency-time and frequency spectrum, with values to be utilised for (1) and (2).

$$
\begin{aligned}
& f_{b}(f)=\frac{f_{b 1}+f_{b 2}}{2} \\
& f_{d}(f)=\frac{f_{b 2}-f_{b 1}}{2}
\end{aligned}
$$

Table 1. FMCW radar simulation properties

\begin{tabular}{cc}
\hline Parameters & Description/Value \\
\hline Waveform type/Sweep method & FMCW/Triangular \\
Sweep bandwidth & $10 \mathrm{MHz}$ \\
Interval band & $20 \mathrm{~ms}$ \\
Sweep period (1 triangular) & \\
Setup 1 (without interval band) & $0 \mathrm{~Hz}-10 \mathrm{MHz}$ \\
Bandwidth 1 (BW1) & $10 \mathrm{~Hz}-20 \mathrm{MHz}$ \\
Betup 2 (with interval band) & \\
Bandwidth 1 (BW1) & $0 \mathrm{~Hz}-10 \mathrm{MHz}$ \\
Bandwidth 2 (BW2) & $12 \mathrm{~Hz}-22 \mathrm{MHz}$ \\
Sampling frequency & $80 \mathrm{MHz}$ \\
Number of sample & 16,00000 \\
Range resolution & $15 \mathrm{~m}$ \\
Target RCS & Swerling $1.10 \mathrm{~m}{ }^{2}$ \\
Target range & $50 \mathrm{~m}$ \\
Target velocity & $60 \mathrm{knots}$ \\
Operating SNR & $-6 \mathrm{~dB}$ to $30 \mathrm{~dB}$ \\
Iteration & 10,000 \\
\hline
\end{tabular}

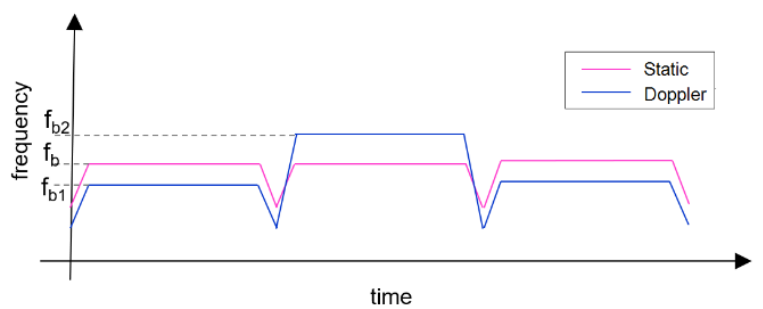

(a)

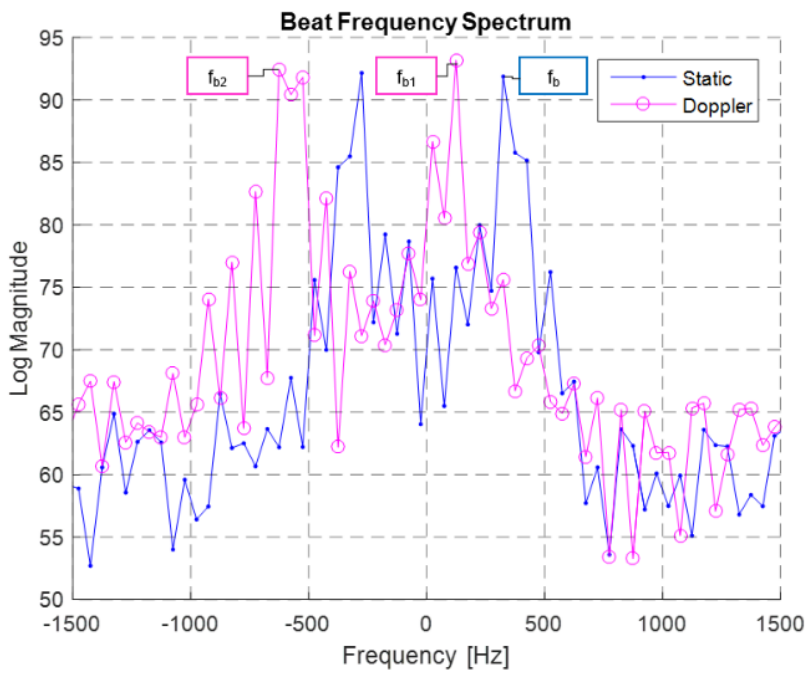

(b)

Figure 3. Example of the beat signal represented in; (a) frequency-time domain, and (b) frequency spectrum 


\section{RESULTS AND DISCUSSION}

\subsection{Received signals spectrogram}

Received signals were observed to have the multi-band effect when no interval band introduced between the two sub-bands. There is frequency overlapping at $10 \mathrm{MHz}$ between BW1 and BW2. The overlapping increases with the existence of doppler caused by the frequency shift due to the target's motion. However, the slight frequency shift by a doppler target is almost unnoticeable through spectrogram display. Figure 4 displays the example of the receiving BW1 spectrogram after bandpass filter for a moving target, without and with interval band implemented. There are remaining BW2 frequency components observed when no interval applied between MIMO sub-bands.

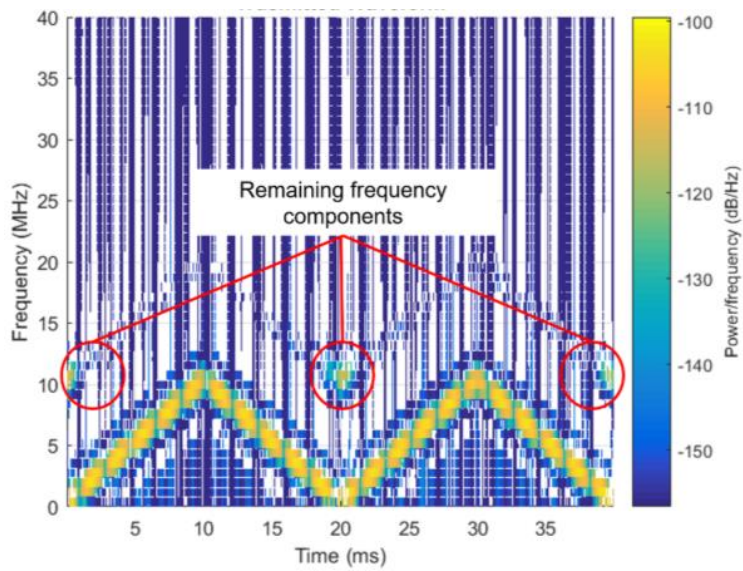

(a)

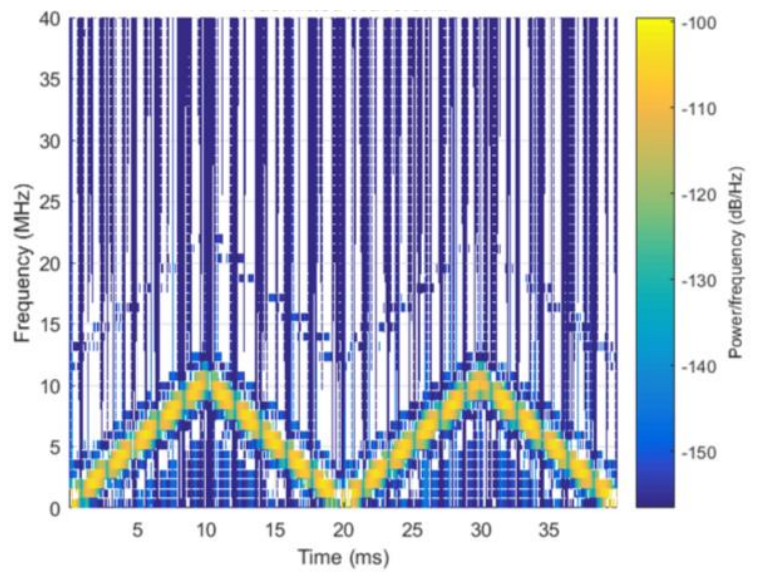

(b)

Figure 4. Spectrogram of receiving signal BW1 after bandpass filter; (a) without interval band implementation, and (b) with $2 \mathrm{MHz}$ interval band implementation, in detecting a moving target

\subsection{SA beat signal frequency spectrum}

The interference effects between two sub-bands was further examined through the beat signal's frequency spectrum. Figure 5 depicts the output of SA taken at $30 \mathrm{~dB}$. Theoretically, $50 \mathrm{~m}$ target's range will result in a beat frequency at approximately $333 \mathrm{~Hz}$ with the waveform's parameters as per Table 1 . The frequency spectrum in Figure 5 displays a static-fluctuating target detection, utilised multi-frequency FMCW with $2 \mathrm{MHz}$ interval band with a positive beat signal of $375 \mathrm{~Hz}$, equivalent to $56.25 \mathrm{~m}$ range. This positive beat frequency is distinct from other peaks for range computation and estimation.

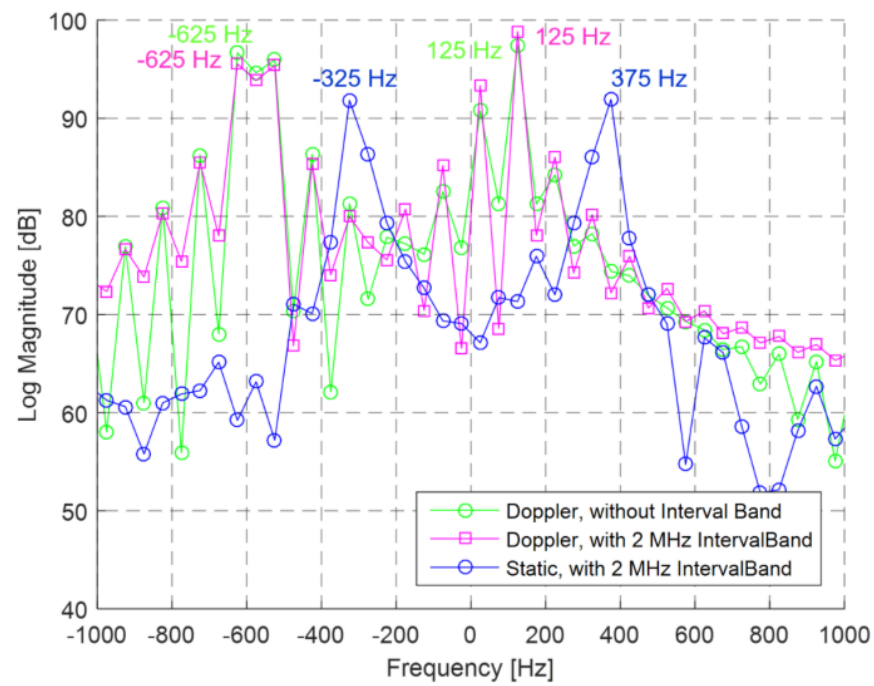

Figure 5. Result of the proposed spectrum averaging frequency spectral, at SNR=30 dB 
However, a doppler-fluctuating target presents a shift of frequencies peaks due to the vessel's motion. It produces a positive peak at $125 \mathrm{~Hz}$ and a negative peak at $-625 \mathrm{~Hz}$, both without and with the interval band approach. Utilising (1) resulted in a beat frequency of $375 \mathrm{~Hz}$ with a corresponding range of $56.25 \mathrm{~m}$. Besides, both spectrums resulted from a doppler target display high neighbouring peaks, which may lead to inaccuracy of range or velocity estimation, in case these peaks overshoot the actual peak.

\subsection{Range error analysis}

The analysis was done on the probability of range error and the average range error. Firstly, the probability of range error was observed over two setups implementing multi-frequency MIMO signals with and without $2 \mathrm{MHz}$ frequency offset in detecting a moving vessel, as illustrated in Figure 6. The exercise is to observe the effect of interval band implementation. In this simulation, a detection was categorised as error when the difference between the actual and estimated range is more than $7.5 \mathrm{~m}$, which is half of the range resolution. By utilising (3), range resolution is equal to $15 \mathrm{~m}$ for $10 \mathrm{MHz}$ sweep bandwidth.

$$
\Delta r=\frac{c}{2 B}
$$

In which $\Delta r$ is range resolution, $c$ is the velocity of light and $B$ is sweep bandwidth. The maximum range error, $\Delta R$, is given by (4) with $T$ is sweep period, $f_{s}$ is the sampling frequency, and $N_{s}$ is the number of samples [17]. From the simulation properties in Table 1, it resulted in the maximum range error of $15 \mathrm{~m}$.

$$
\Delta R=\frac{c T f_{S}}{2 B N_{S}}
$$

Both configurations present along-sided results to each other with a similar curve trend across the simulated SNR. The result indicates that at the $20 \%$ of range error, setup utilising $2 \mathrm{MHz}$ interval displays a better probability of range error performance at approximately $9.14 \mathrm{~dB}$ SNR benefiting from the multifrequency effect and FFT magnitude enhancements by the proposed SA. Configuration without interval band between sub-bands is producing $0.08 \mathrm{~dB}$ delay at $9.22 \mathrm{~dB}$.

Next, an analysis was done over the average range error as depicted in Figure 7, for small moving vessel detection. More significant range error performance is observed between both setup implementation. A configuration with $2 \mathrm{MHz}$ interval surpasses a configuration without interval across SNR. At $26 \mathrm{~dB}$, setup with interval band produces an average range error of $6.06 \mathrm{~m}$ within the error threshold, while arrangement without interval produces a high average error of $23.89 \mathrm{~m}$. However, the average error performance for both setups are still fluctuating and unstable. Setup with and without interval resulted in $6.17 \mathrm{~m}$ of average error at $30 \mathrm{~dB}$, below the $7.5 \mathrm{~m}$ error threshold.

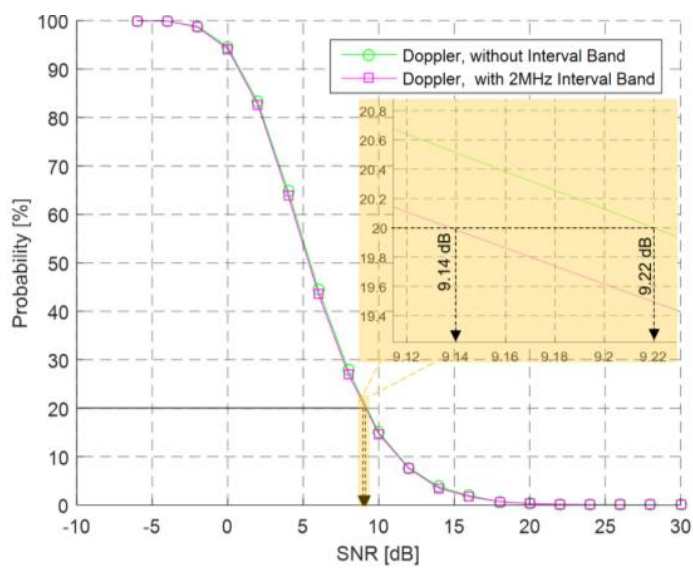

Figure 6. Probability of range error implementing with and without $2 \mathrm{MHz}$ interval between MIMO sub-bands, for small moving vessel detection

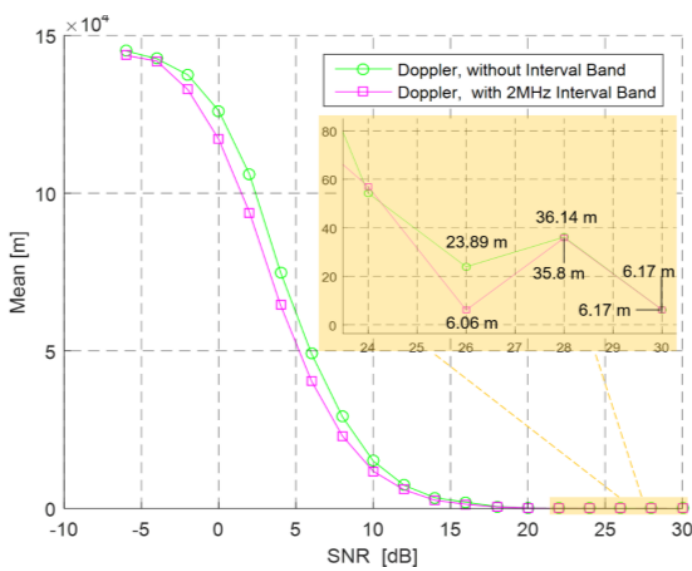

Figure 7. Average range error implementing with and without $2 \mathrm{MHz}$ interval between MIMO subbands, for small moving vessel detection

Following, the range error analysis was also evaluated for configuration with $2 \mathrm{MHz}$ interval band over the vessel's motion, static and moving. It was to discover the effect of vessel motion on the error parameters using the proposed schemes. Figure 8 displays the corresponding probability of range error, while 
Figure 9 displays the average range error. Figure 8 shows that the probability of range error performance in detecting a moving vessel degrades compared to a static vessel, attributable to the doppler's existence that caused the signal to shift in the frequency domain. Vessel motion is known to increase the difficulty in radar detection moreover for low observable target [27], [28] as target RCS depends on targets speed. By utilising a co-located $2 \times 2$ MIMO FMCW radar in detecting a static vessel, the $20 \%$ probability of range error can achieve at $6.35 \mathrm{~dB}$, which is $2.79 \mathrm{~dB}$ better than a moving vessel. It may worsen with the existence of high sea-spikes [29], which was not covered in this simulation.

Similar behaviour is monitored over the average range error for moving and static targets. It shows a similar trend to the probability of range error and resulted in a better error estimation on a small stationary vessel from $26 \mathrm{~dB}$ forward. At $26 \mathrm{~dB}$, the proposed system produces an average range error of $6.06 \mathrm{~m}$ for a moving vessel and $6.25 \mathrm{~m}$ for a vessel without motion. Both readings are within the acceptable error threshold. However, a moving vessel curve fluctuates in performance and reaches $6.17 \mathrm{~m}$ at $30 \mathrm{~dB}$ SNR. Meanwhile, a static target detection continuously displays a smoother curve with a consistent reading of $6.25 \mathrm{~m}$ up until $30 \mathrm{~dB}$.

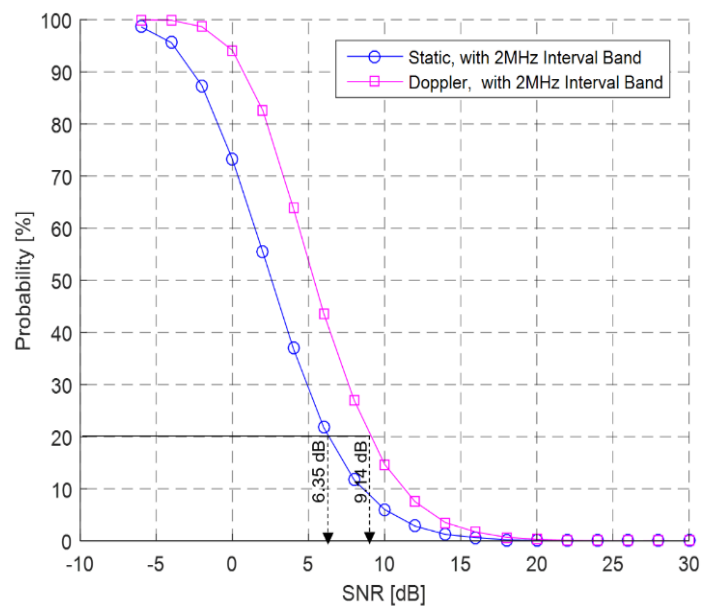

Figure 8. Probability of range error implementing $2 \mathrm{MHz}$ interval between MIMO sub-bands, for small static and moving vessels detection

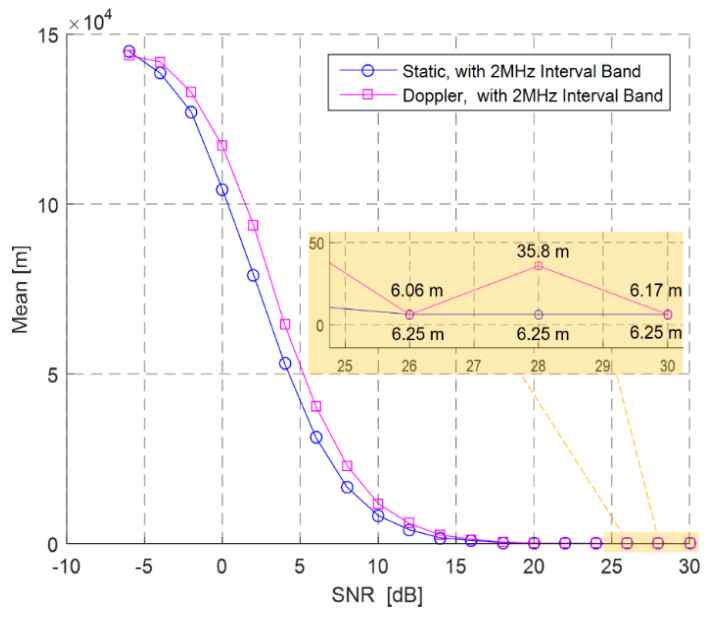

Figure 9. Average range error implementing $2 \mathrm{MHz}$ interval between MIMO sub-bands, for small static and moving vessels detection

\subsection{Velocity error analysis}

Further analysis was done to observe the velocity error. This exercise was to observe the impact of interval band implementation on the velocity error parameters. The velocity error threshold was defined at $5 \mathrm{~ms}^{-1}$ as the maximum velocity error is given by [24].

$$
\Delta V=\frac{c f_{S}}{2 f_{c} N_{S}}
$$

Denotes, $c$ is the velocity of light, $f_{s}$ is the sampling frequency, $f_{c}$ is the carrier frequency and $N_{s}$ is the number of samples.

Figure 10 presents the probability of velocity error in detecting moving vessels using configurations with and without $2 \mathrm{MHz}$ interval. The setup that utilising a $2 \mathrm{MHz}$ gap outplays the setup without gap, against the simulated SNR. At $5.75 \mathrm{~dB}$, the setup with interval already achieved the $20 \%$ of velocity error and followed by setup without interval at $7.5 \mathrm{~dB}$, which is $1.75 \mathrm{~dB}$ latter.

Figure 11 illustrates the average velocity error for both configurations. Similarly, the average velocity error performance displays the design with $2 \mathrm{MHz}$ interval is surpassing the configuration without interval across the SNR. At $24 \mathrm{~dB}$, both setups produce average velocity error within the error threshold, by having $0.58 \mathrm{~ms}^{-1}$ for the setup with interval, and $1.19 \mathrm{~ms}^{-1}$ for the setup without interval. However, the setup without interval is exhibiting unstable readings compare to the other. The setup with interval band is producing average velocity error consistently within the error tolerance. Finally, these two configurations are resulting in an average error below $5 \mathrm{~ms}^{-1}$ at $30 \mathrm{~dB}$. 


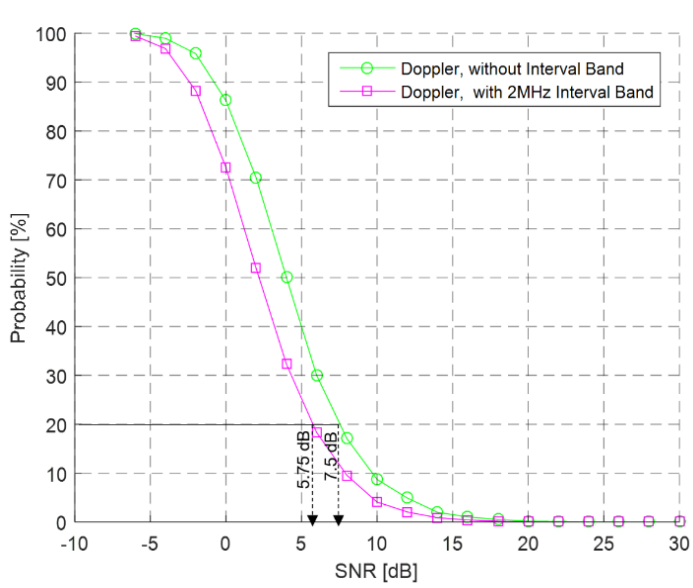

Figure 10. Probability of velocity error implementing with and without $2 \mathrm{MHz}$ interval between MIMO sub-bands, for small moving vessel detection

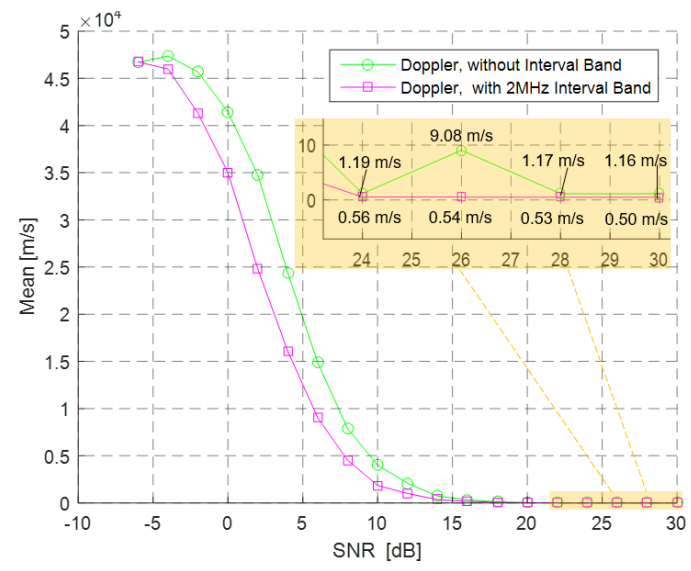

Figure 11. Average velocity error implementing with and without $2 \mathrm{MHz}$ interval between MIMO subbands, for small moving vessel detection

\section{CONCLUSION}

In summary, this paper presented a numerical simulation on a multi-frequency FMCW MIMO radar in detecting a small maritime target. The simulation was done to oversee the proposed configuration of a $2 \times 2$ MIMO radar behaviour in estimation error parameters for a moving vessel by implementing an interval band between MIMO sub-bands and spectrum averaging for combining the received signals. The performance was analysed through the probability of error and average error, for range and velocity. Besides, the receiving signals spectrogram and SA beat frequency spectrum were also examined.

Overall, the multi-frequency MIMO configuration yielded an improvement in performance for range and velocity estimation. It is benefiting from the combination of multiple independent nodes that formed the MIMO configuration. By applying the SA, the received signal's quality is enhanced, and it increases the range estimation accuracy. The proposed scheme surpasses setup without interval band of $0.08 \mathrm{~dB}$ at achieving the $20 \%$ probability of range error and $1.75 \mathrm{~dB}$ at reaching the $20 \%$ probability of velocity error, in doppler target detection. In addition, the average error for range and velocity estimation also indicates the merit brought by MIMO implementation over a maritime target. Furthermore, it was also observed that the existence of a doppler degrades the system's performance in terms of the probability of range error by 2.79 $\mathrm{dB}$ and average range error displays unstable performance, compared to a static vessel. Remaining frequency components exist without interval band implementation. Frequency shifting was observed in the doppler target SA frequency spectral with slightly higher neighboring peaks, which may cause an inaccuracy in estimation if these peaks overshoot the main peaks.

Currently, there is no conclusive data with regards to MIMO radar implementation over maritime small vessel detection. Thus, results gathered from this numerical simulation may lead an important based and a key in designing a multi-frequency FMCW MIMO radar scheme, while improving the estimation error in terms of range and velocity. Further works may include numerical simulation with clutter environment and field experiment validation.

\section{ACKNOWLEDGEMENTS}

The authors grateful acknowledge funding for the study by the Collaborative Research in Engineering, Science and Technology (CREST) under CREST R\&D Grant, project T05C1-67.

\section{REFERENCES}

[1] H. Kabakchiev et al., "Experimental verification of maritime target parameter evaluation in forward scatter maritime radar," IET Radar, Sonar \& Navigation, vol. 9, no. 4, pp. 355-363, 2015, doi:10.1049/iet-rsn.2014.0010.

[2] D. Komorčec and D. Matika, "Small crafts role in maritime traffic and detection by technology integration," Pomorstvo, vol. 30, no. 1, pp. 3-11, 2016, doi: 10.31217/p.30.1.1

[3] International Association of Marine Aids to Navigation and Lighthouse Authorities, "Preparation of Operational and Technical Performance Requirements," $1^{\text {st }}$ ed., no. May, France, 2015.

[4] P. D. L. Williams, H. D. Cramp, and K. Curtis, "Experimental study of the radar cross-section of maritime targets," IEE Journal on Electronic Circuits and Systems, vol. 2, no. 4, pp. 121-136, 1947, doi: 10.1049/ij-ecs.1978.0026. 
[5] I. Harre, "RCS in Radar Range Calculations for Maritime Targets," [Online]. Available: http://www.marit.de/Radar/RCS/RCS_xx.pdf.

[6] J. Suryana and M. Ridha, "Design and implementation of S-Band MIMO FMCW radar," 2016 10th International Conference on Telecommunication Systems Services and Applications (TSSA), 2016, pp. 1-5, doi: 10.1109/TSSA.2016.7871092.

[7] S. Suleymanov, "Design and Implementation of an FMCW Radar Signal Processing Module for Automotive Applications," University of Twente, 2016.

[8] P. Varzakas, "Average channel capacity for Rayleigh fading spread spectrum MIMO systems," International Journal of Communication Systems, vol. 19, no. 10, pp. 1081-1087, 2006, doi: 10.1002/dac.784.

[9] M. I. Skolnik, "Introduction to Radar Systems," New York., vol. 19, pp. 1-539, 1999.

[10] M. I.Skolnik, A. Gupta, M. B. B. S. Frca, C. S. Reilly, "Radar Handbook," 2nd ed. Mc Graw Hill, vol. 24, no. 3, 2011.

[11] Y. P. Saputera, M. Wahab, Y. Y. Maulana, U. I. Nusantara, and B. Batu, "Design of radar display of Indonesian airspace monitoring application," TELKOMNIKA (Telecommunication, Computing, Electronics and Control), vol. 17, no. 3, pp. 1176-1184, 2019, doi: 10.12928/telkomnika.v17i3.11778.

[12] M. S. Kamal and J. Abdullah, "New algorithm for multi targets detection in clutter edge radar environments," Indonesian Journal of Electrical Engineering and Computer Science (IJEECS), vol. 18, no. 1, pp. 420-427, 2020, doi: 10.11591/ijeecs.v18.i1.pp420-427.

[13] J. O. Hinz and U. Zodielzer, "A MIMO FMCW radar approach to HFSWR," Advances in Radio Science, vol. 9, pp. 159-163, 2011, doi: 10.5194/ars-9-159-2011.

[14] J. O. Hinz, T. Fickenscher, A. Gupta, M. Holters, and U. Zolzer, "Evaluation of Time-Staggered MIMO FMCW in HFSWR," $201112^{\text {th }}$ International Radar Symposium (IRS), 2011, pp. 709-713.

[15] X. Chen, B. Chen, Y. Xue, W. Chen, and Y. Huang, "Space-Range-Doppler Focus Processing: A Novel Solution for Moving Target Integration and Estimation Using FDA-MIMO Radar," 2018 International Conference on Radar (RADAR), 2018, pp. 1-4, doi: 10.1109/RADAR.2018.8557297.

[16] P. F. Sammartino, C. J. Baker, and H. D. Griffiths, "Frequency Diverse MIMO Techniques for Radar," IEEE Transactions on Aerospace and Electronic Systems, vol. 49, no. 1, pp. 201-222, 2013, doi: 10.1109/TAES.2013.6404099.

[17] A. Frischen, J. Hasch, C. Waldschmidt, "A Cooperative MIMO Radar Network Using Highly Integrated FMCW Radar Sensors," IEEE Transactions on Microwave Theory and Techniques, vol. 65, no. 4, pp. 1355-1366, 2017, doi: 10.1109/TMTT.2016.2647701.

[18] M. B. Alaie and S. A. Olamaei, "Waveform design for TDM-MIMO radar systems," Signal Processing, vol. 167, 2020, Art. no. 107307, doi: 10.1016/j.sigpro.2019.107307.

[19] S. Zainuddin, I. Pasya, N. E. Abd Rashid, and R. S. Raja Azmir, "Performance of MIMO FMCW Radar in Detecting Small Vessels," 2018 IEEE International RF and Microwave Conference (RFM), 2018, pp. 329-332, doi: 10.1109/RFM.2018.8846475.

[20] H. Gould, J. Tobochnik, and W. Christian, "An Introduction to Computer Simulation Methods-Applications to Physical Systems," 3rd ed. Addison Wesley, 2007.

[21] W. Li, G. Chen, E. Blasch, and R. Lynch, "Cognitive MIMO sonar based robust target detection for harbor and maritime surveillance applications," 2009 IEEE Aerospace conference, 2009, pp. 1-9, doi: 10.1109/AERO.2009.4839469.

[22] P. Koivumäki, "Triangular and Ramp Waveforms in Target Detection with a Frequency Modulated Continuous Wave Radar," Aalto University, 2017.

[23] W. Sediono, "Method of measuring Doppler shift of moving targets using FMCW maritime radar," Proceedings of 2013 IEEE International Conference on Teaching, Assessment and Learning for Engineering (TALE), 2013, pp. 378-381, doi: 10.1109/TALE.2013.6654465.

[24] E. Hyun and J. Lee, "Method to Improve Range and Velocity Error Using De-interleaving and Frequency Interpolation for Automotive FMCW Radars," International Journal of Signal Processing Image Processing and Pattern Recognition, vol. 2, no. 2, pp. 11-22, 2009.

[25] N. Del-Rey-Maestre, D. Mata-moya, M.-P. Jarabo-Amores, P.-J. Gomez-del-Hoyo, and J.-L. Barcena-Humanes, "Artificial intelligence techniques for small boats detection in radar clutter. Real data validation," Engineering Applications of Artificial Intelligence, vol. 67, pp. 296-308, 2018, doi: 10.1016/j.engappai.2017.10.005.

[26] S. Yonemura, A. Tomiki, T. Toda, and T. Kobayashi, "Feasibility of a Radar Altimeter for an Unmanned Aerial Vehicle Cruising in the Mars' Atmosphere," Journal of Selected Areas in Telecommunications (JSAT), vol. 4, no. 2, pp. 23-30, 2014.

[27] X. Yu, X. Chen, Y. Huang, L. Zhang, J. Guan, Y. He, "Radar Moving Target Detection in Clutter Background via Adaptive Dual-Threshold Sparse Fourier Transform," IEEE Access, vol. 7, pp. 58200-58211, 2019, doi: 10.1109/ACCESS.2019.2914232.

[28] X. Chen, "Radon-Fractional Ambiguity Function-Based Detection Method of Low-Observable Maneuvering Target," IEEE Transactions on Aerospace and Electronic Systems, vol. 51, no. 2, pp. 815-833, 2015, doi: 10.1109/TAES.2014.130791.

[29] Y. Wei, L. Guo, and J. Li, "Numerical Simulation and Analysis of the Spiky Sea Clutter from the Sea Surface With Breaking Waves," IEEE Transactions on Antennas and Propagation, vol. 63, no. 11, pp. 4983-4994, 2015, doi: 10.1109/TAP.2015.2476375. 